\title{
The Potential of Dynamic Power and Sub-carrier Assignments in Multi-User OFDM-FDMA Cells
}

\author{
Mathias Bohge, James Gross, Adam Wolisz \\ Telecommunication Networks Group, TU Berlin \\ Einsteinufer 25, 10587 Berlin, Germany \\ \{bohge|gross|wolisz\}@tkn.tu-berlin.de
}

\begin{abstract}
OFDM systems are known to overcome the impairment of the wireless channel by splitting the given system bandwidth into parallel sub-carriers, on which data symbols can be transmitted simultaneously. This enables the possibility of enhancing the system's performance by deploying adaptive (dynamic) mechanisms, namely power and modulation adaption and dynamic sub-carrier assignments. In multi-user communication systems (OFDM-FDMA), these mechanisms can be used to achieve a level of system fairness ensuring that each terminal receives at least an environment-specific minimum amount of data per down-link phase. However, it has been doubted by multiple previous investigations that dynamic power adaption provides enough performance gain in order to be applied in such systems, as it increases the computational load significantly. In this study we discuss the performance gain due to the different approaches and show that in specific communication scenarios enabling a dynamic power distribution provides a significant performance increase compared to dynamic schemes without power adaption.
\end{abstract}

\section{INTRODUCTION}

Future wireless communication systems are expected to deliver high data rates to multiple wireless terminals simultaneously. Due to the impairments of the wireless channel such system will no longer be based on Singlecarrier Modulation $(S C M)$ schemes as the multi-path propagation environment causes wireless channels to be highly frequencyand time selective. Therefore, Orthogonal Frequency Division Multiplexing (OFDM) appears as quite attractive transmission scheme for such future wireless systems: In an OFDM system, the total bandwidth is split into $N$ parallel sub-channels, known as sub-carriers [1], where each sub-carrier can be assumed to experience flat fading.

However, different sub-carriers of a broadband wireless system have a strongly varying attenuation, i.e. the system provides frequency diversity. This diversity can be exploited by dynamically adapting the modulation type and transmit power per sub-carrier at the transmitter, if the sub-carrier attenuations are known. Such dynamic schemes are known as bit loading algorithms [2]-[4] and they provide a significant performance gain (e.g. in terms of throughput) compared to static setups.

In case of a multi-user communication scenario the degree of diversity increases further, as the attenuation of a wireless channel for different terminals is statistically independent.

\footnotetext{
${ }^{1}$ This work has been partially supported by the German research funding agency 'Deutsche Forschungsgemeinschaft (DFG)' under the graduate program 'Graduiertenkolleg 621 (MAGSI/Berlin)'.
}

Thus, in addition to the frequency diversity per terminal an additional multi-user diversity per sub-carrier is present. This fact has lead to the proposal of dynamic resource assignment algorithms in an FDMA fashion. Different terminals are assigned periodically different sets of sub-carriers. Per sub-carrier (again) a different modulation type and a varying amount of power can be employed. Today, many such dynamic sub-carrier assignment algorithms have been proposed [5], [6]. Compared to static schemes these dynamic assignment algorithms can provide a performance increase of $100 \%$ per terminal, simply by utilizing the given bandwidth and transmit power much better.

However, it is much more difficult to optimally assign sets of sub-carriers varying in modulation types and transmit power to the terminals than optimally assigning sets varying in modulation types only. In the latter case each sub-carrier receives an equal amount of transmit power. Together with a target bit error probability the suitable modulation type can be obtained at once. Not so in the case of dynamic power assignments: Here, per sub-carrier multiple different assignment amounts of transmit power (for each modulation type one power value) are possible and the dynamic algorithm has to assign sub-carriers to terminals keeping this additional flexibility in mind. In addition, the total transmit power is limited. Considering the related work, it is known that in the case of bit loading algorithms for wireless point-to-point connections the additional performance obtained from varying the transmit power compared to only applying adaptive modulation is quite small [7], [8], such that it is not recommended to perform dynamic power assignments. In the case of multiuser communications it is an open question if the additional computational burden pays off in terms of performance increase. Several papers claim that adaptive power assignments do not achieve enough additional performance to justify the additional computational cost [9]. In contrast, we show in this paper that the gain obtained from dynamic power assignments is quite significant in certain cases, namely when the average attenuation spread between different terminals in the cell is large enough.

The remainder of this paper is structured as follows: In Section II, we describe our system model. Then, in Section III, we discuss the optimization objective along with the resulting optimization problems. In Section IV, we explain our simulation setup and analysis methodology and then present our 
results. Finally, in Section V, we conclude the paper.

\section{SySTEM MOdEL}

We consider a single cell of a wireless system with radius $r_{\text {cell }}$. Within this cell, one access point coordinates all data transmissions. $J$ terminals are located within the cell. Each terminal continuously downloads data from the access point through the down-link. The terminals are uniformly distributed over the area of the cell. Only the down-link data transmission direction is considered here, using OFDM as transmission scheme. Time is divided into units (frames) of duration $T_{\mathrm{f}}$.

\section{A. Physical layer}

The system features a total bandwidth of $B[\mathrm{~Hz}]$ at center frequency $f_{\mathrm{c}}$. The given bandwidth is split into $N$ subcarriers with a bandwidth of $\frac{B[\mathrm{~Hz}]}{N}$ each [1]. In order to guarantee orthogonality between the sub-carriers, the symbol length is identical for all sub-carriers and it is related to the bandwidth of each sub-carrier by $\frac{N}{B[\mathrm{~Hz}]}=T_{\mathrm{s}}$. Although each sub-carrier employs the same symbol rate, the amount of bits represented by one symbol might be different on each subcarrier. This is due to the deployment of different modulation types (out of a set of $M$ available ones). In addition, a maximum transmit power of $P_{\max }$ is allowed to be radiated by any transmitter of the system. $P_{\max }$ can be arbitrarily split between the sub-carriers.

\section{B. Wireless channel model}

We consider the terminals to be quasi-static and uniformly distributed over the cell. Still, due to the movement of reflecting and scattering objects within the cell, the perceived signal quality per sub-carrier and terminal, i.e., their SNR, varies permanently. The instant SNR of sub-carrier $n$ for terminal $j$ at time $t$ is given by $v_{j, n}^{(t)}=\frac{p_{n}^{(t)} \cdot\left(h_{j, n}^{(t)}\right)^{2}}{\sigma^{2}}$, where $p_{n}^{(t)}$ denotes the transmission power, $h_{j, n}^{(t)}$ denotes the attenuation of subcarrier $n$ and $\sigma^{2}$ denotes the noise power. The attenuation is primarily responsible for the variation of the perceived SNR; it varies due to path loss, shadowing and fading. Thus, $h_{j, n}^{(t)}$ can be decomposed into three factors reflecting these three effects.

The attenuation of each sub-carrier is assumed to be constant over the time unit $T_{\mathrm{f}}$. Note that this time unit is considered to be smaller than the coherence time of the wireless channel, using the definition from [10].

\section{Medium access control layer}

We assume a system where time is divided into frames of length $T_{\mathrm{f}}$. During each frame, a fixed time span is reserved for down-link and up-link transmissions. OFDM-FDMA is applied to the down-link transmissions. Besides reserving a time span for every frame, we do not consider the up-link any further. The duration of one down-link phase is denoted by $T_{\mathrm{d}}$. Prior to each down-link phase, the access point generates new assignments of sub-carrier subsets for each terminal in the cell and/or power assignments for each sub-carrier. Delay effects owing to an inadequate processing power at the access point are not considered. The generation of the

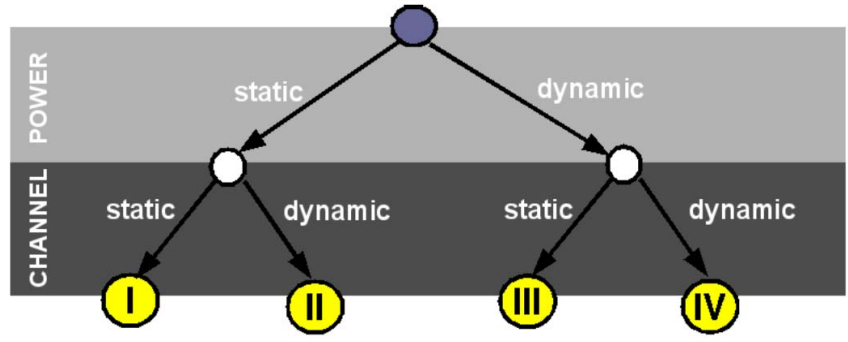

Fig. 1. The four combination possibilities.

new assignments per frame is based on the knowledge of the sub-carrier states towards each terminal denoted by the matrix $\mathbf{H}^{(t)}=\left(h_{j, n}^{(t)} \mid \forall j, n\right)$. It is assumed that this knowledge is available. Note that in a real system this "perfect" channel knowledge will not be available. Instead, the assignments will be based on sub-carrier state estimates with some form of feedback from the terminals to the access point (during the up-link phase). If the length of a frame is chosen sufficiently smaller than the coherence time of the sub-carriers, this channel knowledge will be quite close to the real values during the following frame, i.e. the estimate error will be rather small.

As the access point periodically generates new assignments, the terminals have to be informed about these changes by a signaling system to each payload data transmission. In this work we do not consider the loss due to signaling - thus, we assume the terminals to acquire the knowledge without any performance impact.

\section{STUdiEd APPROACHES}

The system described in Section II features both dynamic assignment mechanisms that were introduced in I: bit loading, and dynamic sub-carrier assignment. Compared to a static assignment scheme, each of the possible combinations of the two schemes (shown in Figure 1) improves the system performance while causing some additional computational effort at the access point. In this section, we precisely characterize each combination by an integer programming problem [11]. The goal of this study is to highlight the performance differences of the optimally solved dynamic combinations, while also considering a static approach.

As described in Section II, each terminal continuously receives a stream of data from the access point (without further specifying the type of data stream). A priori we assume that there is no motivation to give some terminals a higher throughput than others. Therefore, the primary objective is to maximize the minimum throughput of all terminals in the cell, granting each terminal in the cell a minimum average throughput (this approach is sometimes referred to as "rate adaptive" OFDM-FDMA approach [12]). Obviously, the minimum throughput is determined by terminals with the worst attenuation per sub-carrier (referred to as the "weakest" terminals). The dynamic algorithm operates from down-link phase to down-link phase, such that this minimum throughput 
varies from frame to frame, depending on the current subcarrier attenuations.

\section{A. Approach I - Adaptive Modulation with Static Assignments}

We use this case as comparison scheme. For a static FDMA system, each sub-carrier $n$ is always assigned to exactly the same terminal $j$. No reassignment is done. Each terminal gets assigned the same number of sub-carriers $\frac{N}{J}$. The total power $P_{\max }$ - that is radiated by the access point - is equally distributed among all sub-carriers. Depending on the resulting instant SNR $v_{j, n}^{(t)}$ and a target symbol error probability $(S E P)$, for the terminal $j$ on sub-carrier $n$ at the time $t$, the access point chooses the modulation type with the highest possible throughput to be applied (adaptive modulation).

Note that this approach equals a static TDMA scheme with adaptive modulation. Consider [13] for details.

\section{B. Approach II - Equal Power Distribution and Dynamic Sub- carrier Assignment}

In the second approach, we deal with a system that features dynamic sub-carrier assignment in combination with an equal power distribution per sub-carrier. As described in Section II, each sub-carrier $n$ is assigned to at most one terminal $j$ at time $t$, indicated by a binary variable $x_{n, j}^{(t)}$ set to one $\left(x_{n, j}^{(t)}\right.$ is set to zero if $n$ is not assigned to $j$ at $t$ ). Each sub-carrier is employed with equal transmit power. As in approach I, the adaptive modulation scheme is applied on top of the dynamic sub-carrier assignment and fixed power distribution. For the chosen objective, this results in an integer programming problem:

$$
\begin{array}{cl}
\max & \epsilon \\
\text { s.t. } & \sum_{j} x_{j, n}^{(t)} \leq 1 \quad \forall n \\
& \sum_{n} F\left(\frac{p_{n}^{(t)} \cdot h_{j, n}^{(t)}{ }^{2}}{\sigma^{2}}\right) \cdot x_{j, n}^{(t)} \geq \epsilon \quad \forall j,
\end{array}
$$

where $F\left(\frac{p_{n}^{(t)} \cdot h_{j, n}^{(t)}}{\sigma^{2}}\right)$ describes the number of bits per downlink phase that can be transmitted on sub-carrier $n$ for terminal $j$ at time $t$ with a transmit power of $p_{n}^{(t)}$ (thus, this function models the adaptive modulation system, depending also on the target SEP). The first constraint (ALLOC) guarantees the assignment of at most one terminal to one sub-carrier at a time. The second constraint (FAIR) implements the discussed objective: Maximizing the minimum throughput $\epsilon$ per terminal per down-link phase. Note that no additional constraint for the transmit power is required as the power is statically distributed.

\section{Approach III - Dynamic Power Distribution and Static Assignments}

Approach III equals approach I in the static subcarrier/terminal-mapping, yet instead of distributing the power statically over the sub-carriers, it is distributed dynamically with the same objective of maximizing the minimum throughput.

The corresponding optimization problem results as follows:

$$
\begin{array}{ll}
\max & \epsilon \\
\text { s.t. } & \sum_{n} y_{n}^{(t)} \leq P_{\max } \\
& \sum_{n \rightarrow j} F\left(\frac{y_{n}^{(t)} \cdot{h_{j, n}^{(t)}}^{2}}{\sigma^{2}}\right) \geq \epsilon \quad \forall j,
\end{array}
$$

(POWER)

where $n \rightarrow j$ describes a sub-carrier $n$ that is statically assigned to terminal $j$. To point up that the amount of power that is assigned to a terminal $j$ is not a parameter, but the variable of the optimization problem, it is denoted by $y_{n}^{(t)}$. Again, the parameter $h_{j, n}^{(t)}$ is the attenuation that is experienced by terminal $j$ on sub-carrier $n$ at time $t, \sigma^{2}$ is the noise power experienced by each sub-carrier. Hence, the term $y_{n}^{(t)} \cdot h_{j, n}^{(t)}{ }^{2} / \sigma^{2}=v_{n, j}^{(t)}$ describes the instant SNR of terminal $j$ for sub-channel $n . F(\ldots)$ is the functional mapping between the SNR and the amount of bits conveyable per down-link phase. The first constraint (POWER) ensures that the sum of allocated power to the sub-carriers equals or is smaller than the maximum transmission power $P_{\max }$. As in Approach II, the second constraint (FAIR) ensures the maximization of the minimum throughput per frame. Since the sub-carrier assignment is static, there is no need for the sub-carrier allocation constraint (ALLOC) of approach II.

\section{Approach IV - Dynamic Power and Sub-carrier Assignment}

The fourth approach combines both dynamic mechanisms. Hence, both optimization variables $x_{j, n}^{(t)}$ and $y_{n}^{(t)}$ are combined in the optimization problem equation. It is subject to all three constraints that were introduced before:

$$
\begin{array}{ll}
\max & \epsilon \\
\text { s.t. } & \sum_{n} y_{n}^{(t)} \leq P_{\max } \\
& \sum_{j} x_{j, n}^{(t)} \leq 1 \quad \forall n \\
& \sum_{n} F\left(\frac{y_{n}^{(t)} \cdot\left(h_{j, n}^{(t)}\right)^{2}}{\sigma^{2}}\right) \cdot x_{j, n}^{(t)} \geq \epsilon \quad \forall j .
\end{array}
$$

(POWER)

(ALLOC)

Again, $F(\ldots)$ is the functional mapping between the SNR and the amount of bits conveyable per down-link phase, depending on the target SEP.

\section{Performance Analysis}

In this section, we describe the methodology and the scenario setup we assumed when evaluating the four approaches of Section III. We also mention the tools we used to solve the integer programming problems. Finally, we present the results we derived from the simulations. 


\section{A. Methodology and Simulation Scenario}

In order to obtain the system level results, we took the following steps: Initially we generated channel trace files of the attenuation values for each sub-carrier regarding each terminal. One sample was generated for every down-link phase and sub-carrier per terminal. Theses attenuation values were used to generate and solve appropriate instances of the linear programs described in Section III (using the tools ZIMPL $^{2}$ and CPLEX).

When configuring the channel model, we assumed the following scenario. We chose a system with a bandwidth equivalent to IEEE $802.11 \mathrm{a}$, thus the available bandwidth was $B=16.25 \mathrm{MHz}$, split into 48 sub-carriers. As center frequency we chose a channel from the U-NII lower band, located at $5.2 \mathrm{GHz}$. Therefore, the maximum transmit power for this band was $P_{\max }=10 \mathrm{~mW} . J=16$ wireless terminals were located within the cell, uniformly distributed and fixed at their position. The sub-carrier's attenuation changed constantly due to the movement of reflecting objects in the multi-path propagation environment with a maximum speed of $v_{\max }=1 \mathrm{~m} / \mathrm{s}$. The effects influencing the sub-carrier attenuation states were path loss, shadowing and fading. Path loss was determined by the formula $\frac{P_{0}}{P_{\mathrm{tx}}}=K \cdot \frac{1}{d^{\alpha}}$, where $\frac{P_{0}}{P_{\mathrm{tx}}}$ denotes the ratio between received and transmitted power, $d$ denotes the distance between transmitter and receiver, $K$ denotes the reference loss for the distance unit $d$ is measured in and $\alpha$ is the path loss exponent. We parameterized the reference loss with $10 \log (K)=-46.7 \mathrm{~dB}$ and the path loss exponent with $\alpha=2.4$. The shadowing was assumed to be log-normal distributed with a standard deviation of $\sigma=5.8 \mathrm{~dB}$ and a mean of $0 \mathrm{~dB}$ while no correlational behavior was incorporated in the model. For the fading the power spectral density was chosen to have a Jakes-like shape [10] with the Doppler frequency depending on $v_{\max }$ and the center frequency. The multi-path propagation environment was characterized by a delay spread, initially set to $\Delta \sigma=0.15 \mu \mathrm{s}$, with an exponential power delay profile according to the large open space model of ETSI C [14]. An example environment would be a large airport or exposition hall.

For the adaptive modulation, five modulation types were chosen: BPSK, QPSK, 16-QAM, 64-QAM and 256-QAM. In cases of a static power distribution for the resulting SNR the modulation type was chosen which had the highest throughput while still providing a symbol error probability lower than $10^{-2}$. In cases where the power was distributed dynamically, the same symbol error target was applied.

\section{B. Results}

As performance metric we considered the minimum average throughput per cell that could be achieved by solving the optimization problem. The minimum average throughput represents the throughput of the cell that can be guaranteed

\footnotetext{
${ }^{2}$ We gratefully thank Torsten Koch from the Zuse-Institute Berlin (ZIB) for enabling the usage of ZIMPL.
}

by a system provider (or by a system's standard). Thus, it is quite an important metric.

Two different cases were considered. In the first case the radius of the cell was increased (from $10 \mathrm{~m}$ to $200 \mathrm{~m}$ ), leading to a larger difference in the distance between the terminal closest to the access point and the one farest away. Thus, the average attenuation spread (due to the increasing path loss difference of terminals in the cell) of the sub-carriers increased. As the optimization objective is to maximize the minimum throughput for all terminals, the attenuation spread is present in the assigned sub-carriers ${ }^{3}$. In the second case considered, the cell radius was kept fixed (at $100 \mathrm{~m}$ ) but the transmit power was varied (between $0 \mathrm{dBm}$ and $20 \mathrm{dBm}$ ).

All results are given in Figure 2. In the first case (increasing the cell radius) in general the minimum throughput achieved decreases for each approach. The lowest throughput yields the static approach. The best performance is achieved in case of the fully dynamic approach, which is obviously also the case for varying the transmit power. The difference between a fully static approach and the pure power adaption case is rather small (about $300 \mathrm{kBit} / \mathrm{s}$ ), corresponding to results regarding a comparison of adaptive modulation and bit loading in point-to-point connections [7]. For example, spending the computational power in order to perform bit loading at the access point would enable an increase of the cell's radius by about $10 \mathrm{~m}$ for an average minimum throughput of $4 \mathrm{MBit} / \mathrm{s}$ while for an average minimum throughput of $2 \mathrm{MBit} / \mathrm{s}$ the cell could be increased by about $25 \mathrm{~m}$. Switching from the static approach to the dynamic sub-carrier assignment approach yields a larger possible increase: $30 \mathrm{~m}$ in case of the higher minimum throughput and $60 \mathrm{~m}$ in case of the lower one. Notice in particular that the radius gain for switching from the pure dynamic sub-carrier approach to the fully dynamic approach is about $30 \mathrm{~m}$ in case of the higher minimum throughput and $50 \mathrm{~m}$ in case of the lower one. Thus, in particular in the case of the dynamic sub-carrier assignment approach an adaptive power distribution pays off quite well. For a radius of $150 \mathrm{~m}$ switching from the dynamic sub-carrier approach to the fully dynamic approach yields a $700 \mathrm{kBit} / \mathrm{s}$ higher throughput (a gain of 35\%). Hence, especially for cells with a larger radius the fully dynamic approach yields a valuable further performance increase.

This can also be observed from the throughput ratio plot in Figure 2 for an increasing cell radius. In this graph especially the completely different performance gain when switching from a static to a dynamic power distribution for a static subcarrier assignment or for a dynamic sub-carrier assignment is given. While switching from approach 1 to 3 only yields a moderate increase, switching from approach 2 to 4 yields quite a large increase, especially for a large radius.

Also, Figure 2 holds the results for varying the transmit power. In general, as the transmit power increases so does the minimum average throughput for all schemes. As mentioned

\footnotetext{
${ }^{3}$ This is very different to a pure rate maximization approach, where a subcarrier is assigned to the terminal with the lowest attenuation. Thus, distant terminals suffer from starvation.
} 

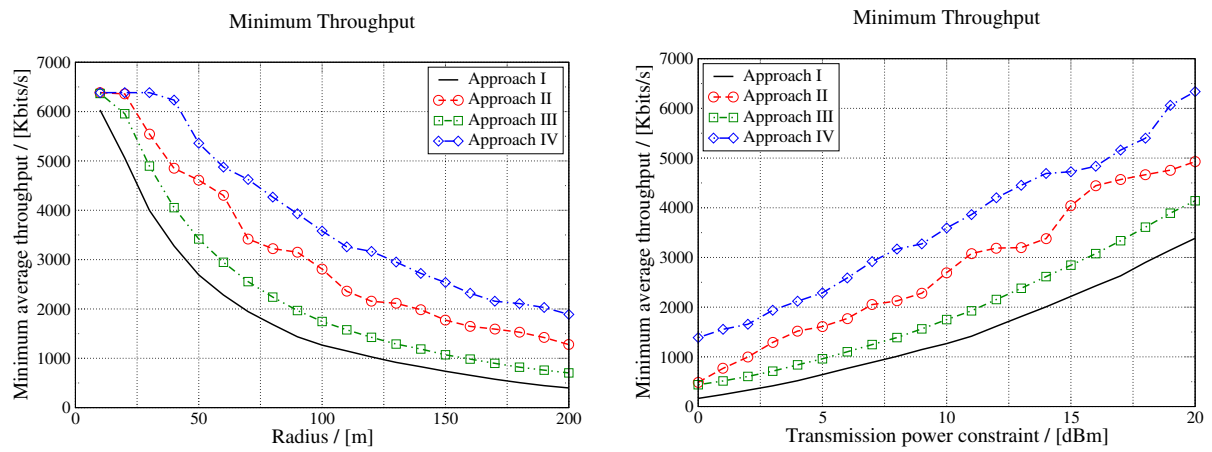

Throughput Ratio
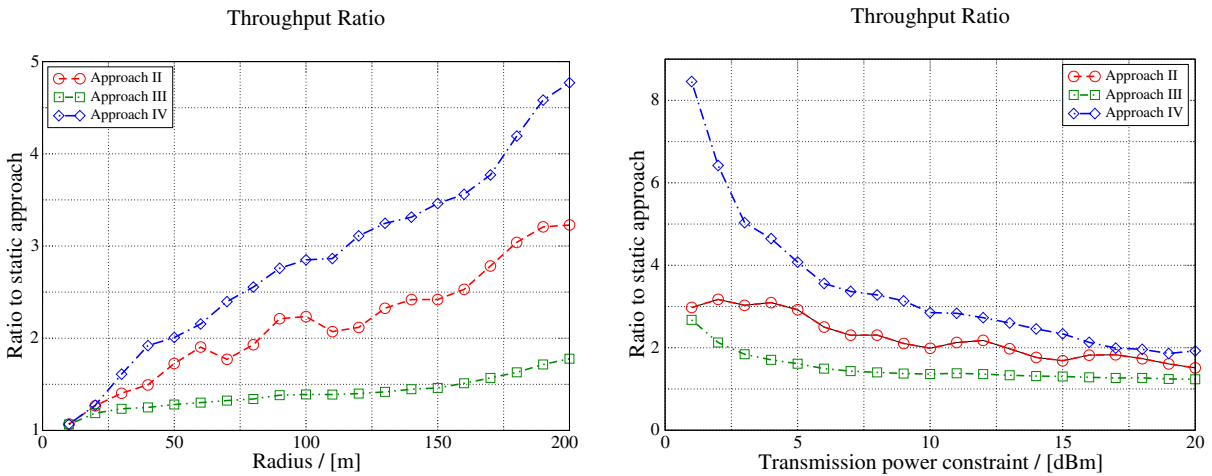

Fig. 2. Comparison of the 4 approaches (I: static system, II: dynamic sub-channel assignment - fixed power, III: pure bit loading, IV: fully dynamic system) regarding the minimum average throughput for an increasing radius (upper left) and transmit power (upper right), and regarding the respective throughput ratios compared to the performance of the static approach (lower left and right)

above, the fully static approach achieves the worst performance and the fully dynamic scheme achieves the best one, while switching from approach 1 to 3 never provides as much additional capacity as switching from approach 2 to 4 .

\section{CONCLUSIONS}

In this study, we compared the most popular adaptive mechanisms that are used to enhance OFDM-FDMA systems, power adaption and dynamic sub-carrier assignment, in terms of the achieved minimum throughput. In contrast to previous investigations we found that the dynamic distribution of power does provide a significant performance increase. This increase is much higher if the sub-carriers are already assigned dynamically. Then, if the transmit power is low or the attenuation spread in the cell is high (thus, a rather large cell is considered) we propose to consider a fully dynamic approach due to the quite high additional performance achieved. In contrast, if only the transmit power is distributed dynamically for a static subcarrier assignment (in FDMA but also in TDMA), the gain is rather small, such that this option is not recommended (in accordance with previous publications).

Regarding the complexity, it is generally suspected that the optimal dynamic sub-carrier assignment following the here presented approach is NP-hard. In contrast, the pure dynamic power distribution can be solved efficiently. Thus, as future work, efficient sub-optimal solution strategies especially for the fully dynamic approach are of interest, since significantly more performance is provided by this approach.

\section{REFERENCES}

[1] R. van Nee and R. Prasad, OFDM Wireless Multimedia Communications, chapter 9, Artech House, 2000.

[2] I. Kalet, "The multitone channel," IEEE Transactions on Communications, vol. 37, no. 2, pp. 119-124, February 1989.

[3] P. Chow, J. Cioffi, and J. Bingham, "A practical discrete multitone transceiver loading algorithm for data transmission over spectrally shaped channels," IEEE Transactions on Communications, vol. 43, no. 2, February 1995.

[4] R. Fischer and J. Huber, "A new loading algorithm for discrete multitone transmission," in IEEE Proc. GLOBECOM, Nov 1996, pp. 724-728.

[5] C.Y. Wong, R.S. Cheng, K.B. Letaief, and R. Murch, "Multiuser OFDM with adaptive subcarrier, bit and power allocation," IEEE Journal on Selected Areas of Comm., vol. 17, no. 10, pp. 1747-1758, Oct 1999.

[6] D. Kivanc and H. Liu, "Subcarrier allocation and power control for OFDMA," in Proc. Conference on Signals, Systems and Computers, 2000, vol. 1, pp. $147-151$

[7] A. Czylwik, "Adaptive OFDM for wideband radio channels," in Proc. of the Global Telecommunications Conf., 1996, vol. 2, pp. 713 - 718.

[8] T. Hunziker and D. Dahlhaus, "Optimal Power Adaptation for OFDM Systems with Ideal Bit-Interleaving and Hard-Decision Decoding," in Proc. IEEE Int. Conference on Communications (ICC), 2003, vol. 5, pp. $3392-3397$

[9] W. Rhee and J. Cioffi, "Increase in capacity of multiuser OFDM system using dynamic subchannel allocation," in Proc. Vehicular Technology Conference (VTC), 2000, pp. 1085 - 1089.

[10] J. Cavers, Mobile Channel Characteristics, chapter 1.3, Kluwer Academic, 2000.

[11] A. Schrijver, Combinatorial Optimization, Springer, 2003.

[12] D. Kivanc, G. Li, and H. Liu, "Computationally efficient bandwidth allocation and power control for OFDMA," IEEE Transactions on Wireless Communications, vol. 2, no. 6, pp. 1150-1158, 2003.

[13] M. Bohge, "Bit loading versus dynamic sub-carrier assignment in multiuser OFDM-FDMA systems," September 2004, Diploma Thesis a Technical University of Berlin, Germany.

[14] J. Medbo and P. Schramm, Channel Models for HIPERLAN/2, ETSI EP BRAN document 3ERI085B, March 1998. 